\title{
Thin and dressed Polyakov loops from spectral sums of lattice differential operators
}

\section{Christian Hagen* and Falk Bruckmann}

Institut für Theoretische Physik, Universität Regensburg, D-93040 Regensburg, Germany

\section{Erek Bilgici* and Christof Gattringer}

Institut für Physik, FB Theoretische Physik, Universität Graz, A-8010 Graz, Austria

E-mails: erek.bilgici@uni-graz.at, falk.bruckmann@physik.uni-r.de, christof.gattringer@uni-graz.at, christian.hagenephysik.uni-r.de

\begin{abstract}
We represent thin and dressed Polyakov loops as spectral sums of eigenvalues of differential operators on the lattice. For that purpose we calculate complete sets of eigenvalues of the staggered Dirac and the covariant Laplace operator for several temporal boundary conditions. The spectra from different boundary conditions can be combined such that they represent single (thin) Polyakov loops, or a collection of loops (dressed Polyakov loops). We analyze the role of the eigenvalues in the spectral sums below and above the critical temperature.
\end{abstract}

The XXV International Symposium on Lattice Field Theory July 30 - August 42007

Regensburg, Germany

*Based on presentations by Erek Bilgici and Christian Hagen. 


\section{Motivation}

The phenomenology of QCD is governed by two prominent features, confinement and spontaneous breaking of chiral symmetry. As one increases the temperature $T$ above some critical value $T_{c}$, the theory becomes deconfined and chiral symmetry is restored. This suggests that there could be a relation between the two phenomena. Establishing or ruling out such a relation would be a major insight into key mechanisms of QCD.

The finite temperature transition of pure gauge theory, where the system changes from the confined $\left(T<T_{c}\right)$ into the deconfined phase $\left(T>T_{c}\right)$, can be understood as spontaneous breaking of the center symmetry [1], and the Polyakov loop is a suitable order parameter with $\langle P\rangle=0$ for $T<T_{c}$ and $\langle P\rangle \neq 0$ for $T>T_{c}$ (see Fig. 1). Concerning chiral symmetry breaking the order parameter is given by the chiral condensate with $\langle\bar{\psi} \psi\rangle \neq 0$ for $T<T_{c}$ and $\langle\bar{\psi} \psi\rangle=0$ for $T>T_{c}$. The chiral condensate in turn is related to the spectral density of the Dirac operator by the BanksCasher formula [2]: $\langle\bar{\psi} \psi\rangle=-\pi \rho(0)$, where $\rho(0)$ is the spectral density at the origin. When increasing the temperature above the critical value, the spectral density at the origin vanishes and with it the chiral condensate (see Fig. 2).

In a series of recent papers, [3]-[6], the Polyakov loop, or other quantities that serve as order parameters for the breaking of the center symmetry, were related to spectral sums of differential operators on the lattice, in particular the Dirac and the covariant Laplace operators. In this way also confinement is related to spectral quantities, as is chiral symmetry breaking through the Banks Casher formula. Since eigenvalues can be divided into IR and UV regions in a natural way, the spectral representations allow one to analyze whether confinement is dominated by IR or UV modes. In this paper we review and extend our contributions to this enterprise.

\section{Derivation of spectral sums}

\subsection{Preliminaries}

The thin Polyakov loop averaged over space is given by

$$
P=\frac{1}{V_{3}} \sum_{\vec{x}} L(\vec{x}) \quad \text { with } \quad L(\vec{x})=\operatorname{Tr}_{c}\left[\prod_{x_{4}=1}^{N_{t}} U_{4}\left(\vec{x}, x_{4}\right)\right]
$$

where $L(\vec{x})$ is a straight line of links in temporal direction closed around the periodic boundary and $\operatorname{Tr}_{c}$ denotes the color trace. Obviously, this quantity has a rather singular support. Due to this fact it is known to have a poor continuum limit with large renormalization effects.

To address this problem, below we will define a new observable $P^{(q)}$ which we refer to as the dressed Polyakov loop with winding number $q$. It is a collection of loops all with the same number $q$ of windings around compactified time. So, for example, $P^{(1)}$ is a sum of the thin Polyakov loop and all other loops winding once around the lattice in time direction, where the latter loops may have arbitrary complicated detours in spatial directions and are weighted proportional to their length.

In the following two sections we derive the spectral sums for thin and dressed Polyakov loops. For this purpose it is convenient to summarize our conventions: The staggered Dirac operator is 

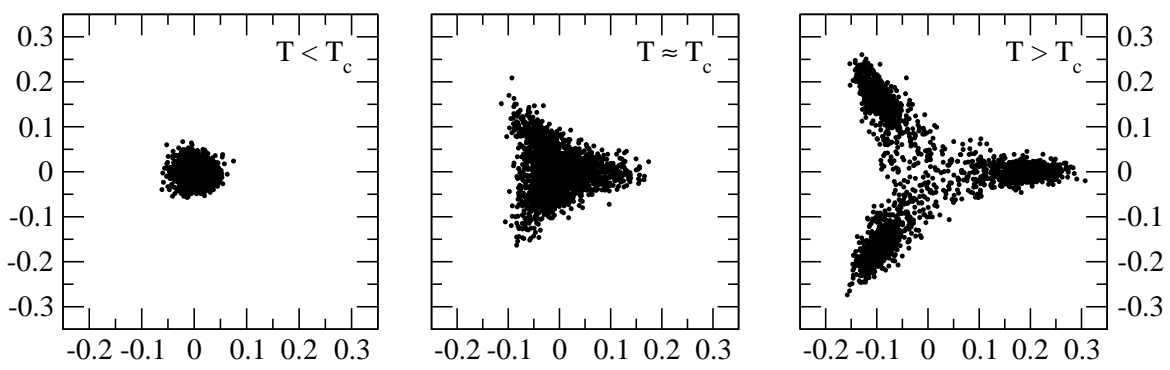

Figure 1: Scatter plot for the thin Polyakov loop in the complex plane for an ensemble of quenched SU(3) configurations. As the temperature increases (left to right) the Polyakov loop acquires a non-vanishing expectation value.
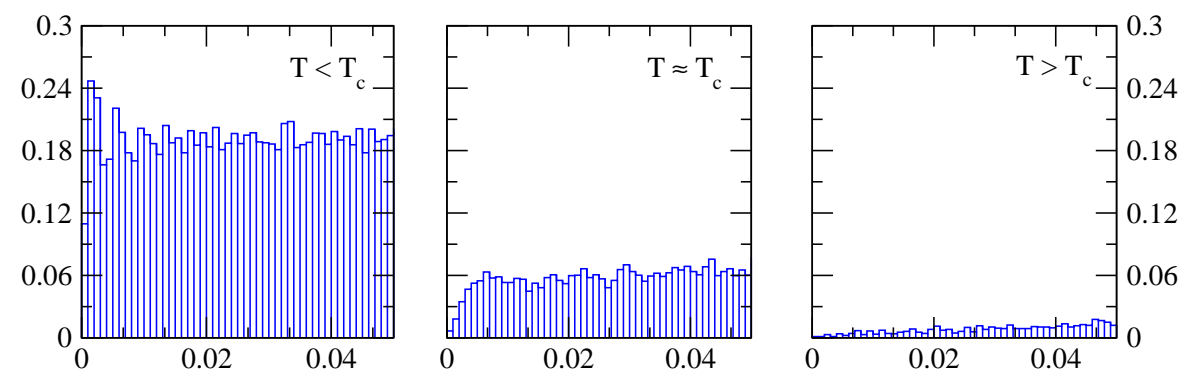

Figure 2: Histograms of the spectral density of the staggered lattice Dirac operator as a function of the modulus of the eigenvalues. Below the critical temperature the spectral density at the origin is non-zero. Near $T_{c}$ the density at the origin decreases and vanishes above the critical temperature.

given by

$$
D(x, y)=m \delta_{x, y}+\frac{1}{2} \sum_{\mu=1}^{4} \eta_{\mu}(x)\left[U_{\mu}(x) \delta_{x+\hat{\mu}, y}-U_{\mu}(x-\hat{\mu})^{\dagger} \delta_{x-\hat{\mu}, y}\right]
$$

where $\eta_{\mu}(x)=(-1)^{x_{1}+x_{2}+\ldots+x_{\mu-1}}$ is the staggered phase. The covariant Laplace operator on the lattice is defined as

$$
\Delta(x, y)=\left(8+m^{2}\right) \delta_{x, y}-\sum_{\mu=1}^{4}\left[U_{\mu}(x) \delta_{x+\hat{\mu}, y}+U_{\mu}(x-\hat{\mu})^{\dagger} \delta_{x-\hat{\mu}, y}\right] .
$$

Below we will use the staggered Dirac operator for representing the thin Polyakov loop, while the dressed loops are constructed from the Laplace operator. We stress, however, that it is an easy exercise to generalize our formulas, such that both the thin and the dressed Polyakov loops can be obtained from both differential operators. The derivation can be extended further to arbitrary lattice operators with only nearest neighbor interaction (such as Domain Wall Fermions). The advantage of these operators is that they are numerically cheap. 


\subsection{Thin Polyakov loops}

In order to connect the thin Polyakov loop to spectral sums we follow the derivation in [3]. We consider powers of the staggered Dirac operator (2.2). The $n$-th power of the operator then contains paths up to length $n$, dressed with a product of $n$ links. For the special case where we consider the operator to the power $N_{t}$, where $N_{t}$ is the time extent of our lattice, we obtain

$$
\begin{aligned}
\operatorname{Tr}_{c} D^{N_{t}}(x, x)= & \frac{1}{2^{N_{t}}} \operatorname{Tr}_{c} \prod_{s=1}^{N_{t}} U_{4}(\vec{x}, s)-\frac{1}{2^{N_{t}}} \operatorname{Tr}_{c} \prod_{s=0}^{N_{t}-1} U_{4}\left(\vec{x}, N_{t}-s\right)^{\dagger} \\
& + \text { other loops, trivially closed } \\
= & \frac{1}{2^{N_{t}}}\left[L(\vec{x})-L^{*}(\vec{x})\right]+\text { other loops, trivially closed. }
\end{aligned}
$$

From the set of all loops that contribute we have singled out the thin Polyakov loop and its complex conjugate. These two straight loops are the only ones that can close around compactified time. All others are 'trivially closed', i.e., they do not wrap around the lattice in temporal direction.

The key observation is that a change of the temporal boundary conditions

$$
U_{4}\left(\vec{x}, N_{t}\right) \rightarrow z U_{4}\left(\vec{x}, N_{t}\right), \quad|z|=1,
$$

where $z$ is a complex phase, only affects the Polyakov loops

$$
L \longrightarrow z L
$$

while the trivially closed loops remain unchanged. After such a change the expression (2.5) reads

$$
\operatorname{Tr}_{c} D_{z}^{N_{t}}(x, x)=\frac{1}{2^{N_{t}}}\left[z L(\vec{x})-z^{*} L^{*}(\vec{x})\right]+\text { other loops, }
$$

where $D_{z}$ is the Dirac operator on a configuration where the temporal boundary conditions are changed by a factor $z$. Averaging this expression over space and time we obtain

$$
\operatorname{Tr} D_{z}^{N_{t}}=\frac{V_{4}}{2^{N_{t}}}\left(z P-z^{*} P^{*}+X\right)
$$

where $X$ is the sum of all trivially closed paths. We make use of this behavior to cancel the unwanted trivial contributions and project onto the thin Polyakov loop by taking linear combinations (coefficients $a_{i}$ ) of this expression for three different boundary conditions $z_{1}, z_{2}$ and $z_{3}$

$$
\begin{aligned}
P & \stackrel{!}{=} 2^{N_{t}} \sum_{i=1}^{3} a_{i} \operatorname{Tr}\left(D_{z_{i}}^{N_{t}}\right) \\
& =P \underbrace{\left(z_{1} a_{1}+z_{2} a_{2}+z_{3} a_{3}\right)}_{\stackrel{!}{=} 1}-P^{*} \underbrace{\left(z_{1}^{*} a_{1}+z_{2}^{*} a_{2}+z_{3}^{*} a_{3}\right)}_{\underbrace{*} 0}+X \underbrace{\left(a_{1}+a_{2}+a_{3}\right)}_{\stackrel{!}{=} 0} .
\end{aligned}
$$

This leads to a set of linear equations for the coefficients $a_{1}, a_{2}$ and $a_{3}$

$$
\left(\begin{array}{ccc}
z_{1} & z_{2} & z_{3} \\
z_{1}^{*} & z_{2}^{*} & z_{3}^{*} \\
1 & 1 & 1
\end{array}\right)\left(\begin{array}{l}
a_{1} \\
a_{2} \\
a_{3}
\end{array}\right)=\left(\begin{array}{l}
1 \\
0 \\
0
\end{array}\right) .
$$


One can show that this equation has a unique solution as long as the boundary conditions $z_{1}, z_{2}$ and $z_{3}$ are different. For the particular choice $z_{1}=1$ and $z_{2}=z_{3}^{*}=e^{i \frac{2 \pi}{3}} \equiv z$ the thin Polyakov loop is then given by

$$
P=\frac{2^{N_{t}}}{V_{4}}\left[\sum_{i}\left(\lambda^{(i)}\right)^{N_{t}}+z^{*} \sum_{i}\left(\lambda_{z}^{(i)}\right)^{N_{t}}+z \sum_{i}\left(\lambda_{z^{*}}^{(i)}\right)^{N_{t}}\right],
$$

where the $\lambda_{z}^{(i)}$ are the eigenvalues of the staggered Dirac operator for a given boundary condition $z$. Similar expressions can be obtained for other differential operators on the lattice.

\subsection{Dressed Polyakov loops}

In order to obtain the spectral sum for the dressed Polyakov loops we now use the Laplace operator and apply a different strategy than used for the thin loops. First, we rewrite the covariant Laplace operator on the lattice from Eq. (2.3) in the following way

$$
\Delta=\frac{1}{\kappa}[\mathbb{1}-\kappa H], \quad H(x, y)=\sum_{\mu=1}^{4}\left[U_{\mu}(x) \delta_{x+\hat{\mu}, y}+U_{\mu}(x-\hat{\mu})^{\dagger} \delta_{x-\hat{\mu}, y}\right],
$$

where the hopping matrix $H$ collects all terms which connect nearest neighbors on the lattice and the hopping parameter $\kappa$ is related to the bare mass $m$ via $\kappa=\left(8+m^{2}\right)^{-1}$. The inverse Laplace operator $\Delta^{-1}(x, x)$ can be expressed as geometric series in terms of powers of $H$,

$$
\Delta^{-1}(x, x)=\sum_{j=0}^{\infty} \kappa^{j+1} H^{j}(x, x) .
$$

Since the hopping matrix $H$ contains only terms that connect nearest neighbors, the powers $H^{j}(x, x)$ in (2.14) correspond to closed loops of length $j$, again dressed with the corresponding products of the link variables. Thus we can organize $\Delta^{-1}$ in terms of loops and order these with respect to their winding around time. We obtain (after taking the trace)

$$
\operatorname{Tr} \Delta^{-1}=\sum_{x} \operatorname{Tr}_{c} \Delta^{-1}(x, x)=\sum_{n \in \mathbb{Z}} \sum_{l \in \mathscr{L}_{n}} \kappa^{|l|+1} \operatorname{Tr}_{c} \prod_{(y, \mu) \in l} U_{\mu}(y)
$$

where $\mathscr{L}_{n}$ is the set of loops that wind exactly $n$-times around the time direction and $|l|$ denotes the length of a loop $l$. It is important that now the sum on the right hand side contains loops of arbitrary length, with all possible numbers of windings. To disentangle the different winding numbers we introduce $\mathrm{U}(1)$-valued temporal boundary conditions for the Laplace operator which are most conveniently implemented by the replacement

$$
U_{4}\left(\vec{x}, N_{t}\right) \longrightarrow e^{i \varphi} U_{4}\left(\vec{x}, N_{t}\right)
$$

which corresponds to a parameterization $z=e^{i \varphi}$ in (2.6). Each loop acquires a phase factor $e^{i \varphi n}$, corresponding to its winding number $n$ :

$$
\operatorname{Tr}\left(\Delta_{\varphi}\right)^{-1}=\sum_{n \in \mathbb{Z}} e^{i \varphi n} \sum_{l \in \mathscr{L}_{n}} \kappa^{|l|+1} \operatorname{Tr}_{c} \prod_{(y, \mu) \in l} U_{\mu}(y) .
$$


Integrating over $\varphi$ with a factor of $e^{-i \varphi q}$ projects to winding number $q$ and we end up with the dressed Polyakov loop $P^{(q)}$ (which we normalize with $V=N^{3} N_{t}$ ),

$$
P^{(q)} \equiv \frac{1}{V} \int_{-\pi}^{\pi} \frac{d \varphi}{2 \pi} e^{-i \varphi q} \operatorname{Tr}\left(\Delta_{\varphi}\right)^{-1}=\frac{1}{V} \sum_{l \in \mathscr{L}_{q}} \kappa^{|l|+1} \operatorname{Tr}_{c} \prod_{(y, \mu) \in l} U_{\mu}(y)
$$

After the projection the loops still can be arbitrarily long but all have the same winding number $q$. The individual loops are weighted with a factor $\kappa^{|l|+1}$, where $|l|$ is the length of the loop. This leads to an exponential suppression of long loops and the rate of the suppression is determined by the value of $\kappa$. Nevertheless arbitrary long loops may contribute and the formula (2.18) is a sensible definition of dressed loops.

The final step to make the connection to spectral properties of the staggered Dirac operator is to write the trace over $\Delta^{-1}$ as a spectral sum giving

$$
P^{(q)}=\frac{1}{V} \int_{-\pi}^{\pi} \frac{d \varphi}{2 \pi} e^{-i \varphi q} \sum_{j=1}^{3 V} \frac{\kappa}{1-\kappa \lambda_{j}^{(\varphi)}}=\frac{1}{V} \sum_{l \in \mathscr{L}_{q}} \kappa^{|l|+1} \operatorname{Tr}_{c} \prod_{(y, \mu) \in l} U_{\mu}(y),
$$

where $\lambda_{j}^{(\varphi)}$ is the $j$-th eigenvalue of the hopping matrix $H$ (2.13) for boundary condition angle $\varphi$. The dressed Polyakov loop transforms in the same way as the thin Polyakov loop. Thus the dressed Polyakov loops are proper order parameters for the breaking of the center symmetry. They are also not as singular as the thin Polyakov loops and thus are expected to have a better continuum limit with smaller renormalization effects. In an analogous way dressed Polyakov loops can be constructed also with the eigenvalues of the Dirac operator instead of the Laplace spectrum.

We stress that both equations (2.12) and (2.19) are exact results that hold for individual gauge configurations. However, for a practical evaluation with numerically generated gauge configurations the two formulas have a different status. Equation (2.12) for the thin loops holds to machine precision when the result of the spectral sum is compared to the direct evaluation of the thin Polyakov loop from the gluonic definition (2.1). The expression (2.19) for the dressed loop on the other hand contains an integral over a continuum of boundary conditions. That this integral can indeed be approximated numerically in a sensible way will be demonstrated in the next section.

\begin{tabular}{|c|c|c|c|}
\hline$\beta$ & $L^{3} \times N_{t}$ & $\mathrm{a}[\mathrm{fm}]$ & $\mathrm{T}[\mathrm{MeV}]$ \\
\hline \hline 7.00 & $6^{3} \times 4,12^{3} \times 4$ & $0.351(3)$ & 140 \\
\hline 7.60 & $6^{3} \times 4,12^{3} \times 4$ & $0.194(4)$ & 254 \\
\hline 7.91 & $6^{3} \times 4,12^{3} \times 4$ & $0.146(2)$ & 336 \\
\hline \hline 7.40 & $12^{3} \times 6$ & $0.234(2)$ & 140 \\
\hline 8.06 & $12^{3} \times 6$ & $0.129(1)$ & 255 \\
\hline 8.40 & $12^{3} \times 6$ & $0.098(1)$ & 337 \\
\hline
\end{tabular}

Table 1: Ensembles used in our simulations. For the $6^{3} \times 4$ lattices 2000 configurations are available, for $12^{3} \times 4$ and $12^{3} \times 6$ we have used 20 configurations. 


\section{Numerical analysis}

\subsection{Simulation details}

For the numerical analysis of our spectral sums (2.12) and (2.19) we use quenched $S U(3)$ gauge configurations generated with the Lüscher-Weisz action [7]. We work on lattices with different sizes ranging from $6^{3} \times 4$ up to $12^{3} \times 6$ at temperatures below and above the critical value $T_{c}$ (see Table 1). For those we compute complete spectra of both the staggered Dirac operator and the hopping matrix $H$ of the Laplace operator for up to 16 different boundary condition. The lattice spacing was set [8] with the Sommer parameter $r_{0}=0.5 \mathrm{fm}$. All error bars we show are statistical errors determined with single elimination jackknife.

\subsection{Thin Polyakov loops}

For the thin Polyakov loops we restrict ourselves to the three boundary conditions $z_{1}=1$ and $z_{2}=z_{3}^{*}=e^{i \frac{2 \pi}{3}} \equiv z$ which are used to derive the spectral sum (2.12). A different choice would change our results only marginally.

After computing all eigenvalues of the staggered Dirac operators we order them w.r.t. to their absolute value and organize them in bins. For each bin we calculate a number of observables. The first observable is the distribution of the eigenvalues, which we show in Fig. 3 for different spatial and temporal extents of the lattice and for three different temperatures above, below and approximately at the critical value $T_{c}$. The l.h.s. set of those plots shows the distribution as a function of the size of the eigenvalues in lattice units, while the r.h.s. plots are in MeV. One can clearly see that the spectral density at the origin vanishes and chiral symmetry is restored as one increases the temperature above the critical value. The density of eigenvalues reaches a maximum after about three quarters of the eigenvalues and then quickly drops towards the UV cutoff.

Fig. 3 is particularly important when one analyzes which part of the spectrum contributes to the spectral sum (2.12) for the thin Polyakov loop. For the interpretation of such an analysis it is necessary to take into account the global distribution of the eigenvalues, and this is exactly what Fig. 3 provides.

In the spectral sum (2.12) the thin Polyakov loop emerges through a shift of the eigenvalues as the boundary condition is changed. It is interesting to analyze how strongly different eigenvalues of the spectrum are shifted when the boundary condition is changed. We quantify this question by studying the averaged shift of the eigenvalues given by

$$
s(\lambda)=\left(\left|\lambda-\lambda_{z}\right|+\left|\lambda-\lambda_{z^{*}}\right|+\left|\lambda_{z}-\lambda_{z^{*}}\right|\right) / 3 .
$$

Since above the phase transition the thin Polyakov loop obtains a finite value while in the confined phase it is approximately zero, the shift of the eigenvalues should change as one increases the temperature. This is exactly what we find in our results for the average shift $s(\lambda)$ presented in Fig. 4). The individual data points are obtained by averaging over all eigenvalues in a bin and then over all configurations in the ensemble.

It is obvious, that over the whole range of the spectrum the average shift is almost zero below the critical temperature. For high temperatures, however, we find large shifts for low-lying eigenvalues and little or no shift when the UV region is approached. The small shift at the UV end of the spectrum disappears when finer and larger lattices are used. 

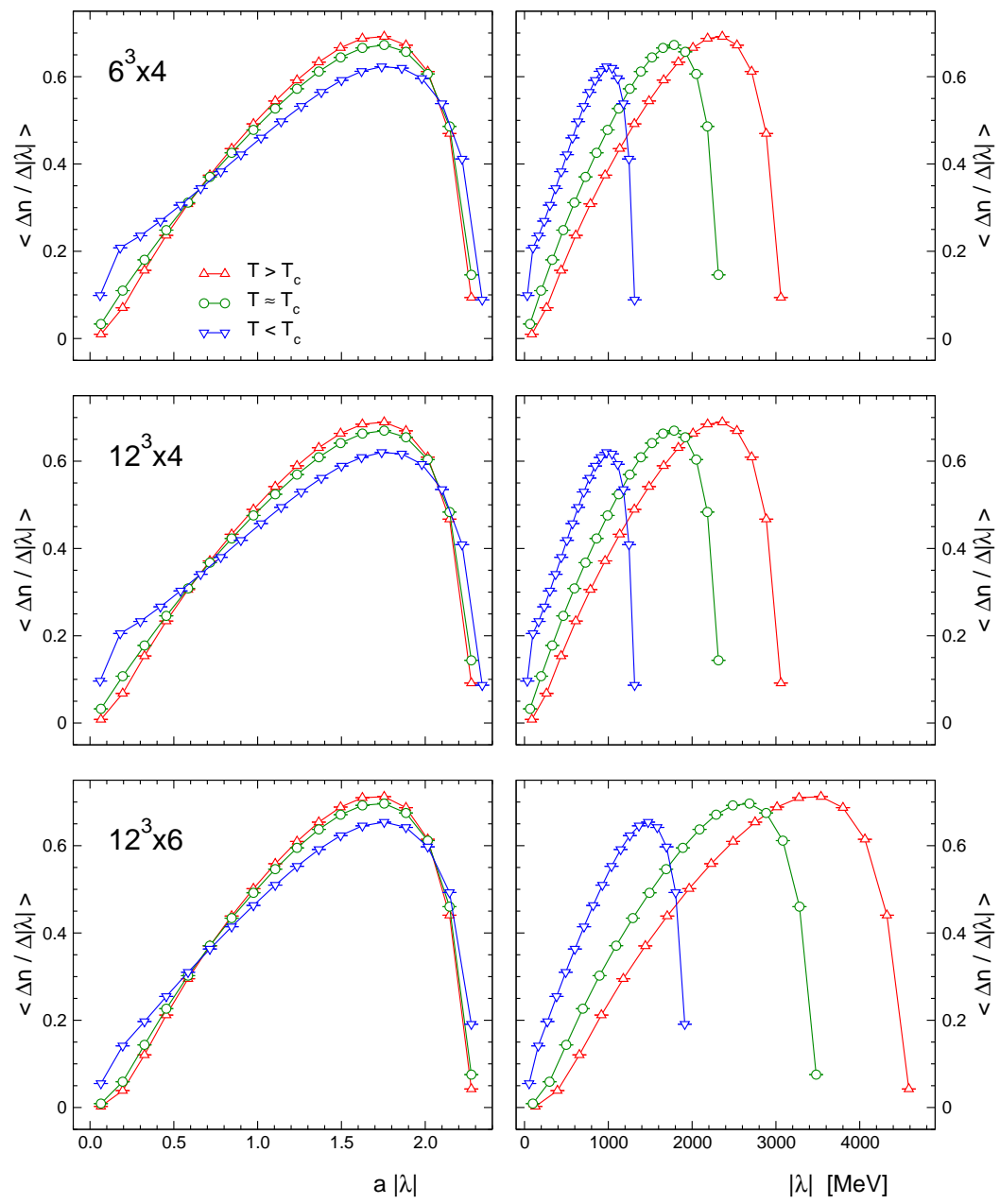

Figure 3: Distribution of the eigenvalues of the staggered Dirac operator for different lattices and different temperatures. The 1.h.s. plots are in lattice units, while on the r.h.s. we use MeV.

After understanding the global distribution of the eigenvalues and their shift under a change of the boundary conditions, we can start to analyze the individual contributions to the spectral sum (2.12). This individual contribution is given by

$$
c(\lambda)=\frac{2^{N_{t}}}{V_{4}}\left[(\lambda)^{N_{t}}+z^{*}\left(\lambda_{z}\right)^{N_{t}}+z\left(\lambda_{z^{*}}\right)^{N_{t}}\right] .
$$

Our results are shown in Fig. 5, where we plot the absolute value of the individual contributions after normalizing them with $P$, i.e., we divide by the value of the thin Polyakov loop. It is obvious that mainly the UV modes contribute to the thin Polyakov loop. This observation is essentially independent of the temperature and the size of the lattice. This is a finding which is not a-priori obvious, given the fact that it is the IR modes that show the largest shifts in Fig. 4. On the other hand, the large power $N_{t}$ in the spectral sum (2.12) drastically enhances the contributions of the UV eigenvalues.

Interesting are also the dips which form with increasing temperature at about $1.5 a$ for $N_{t}=4$ 

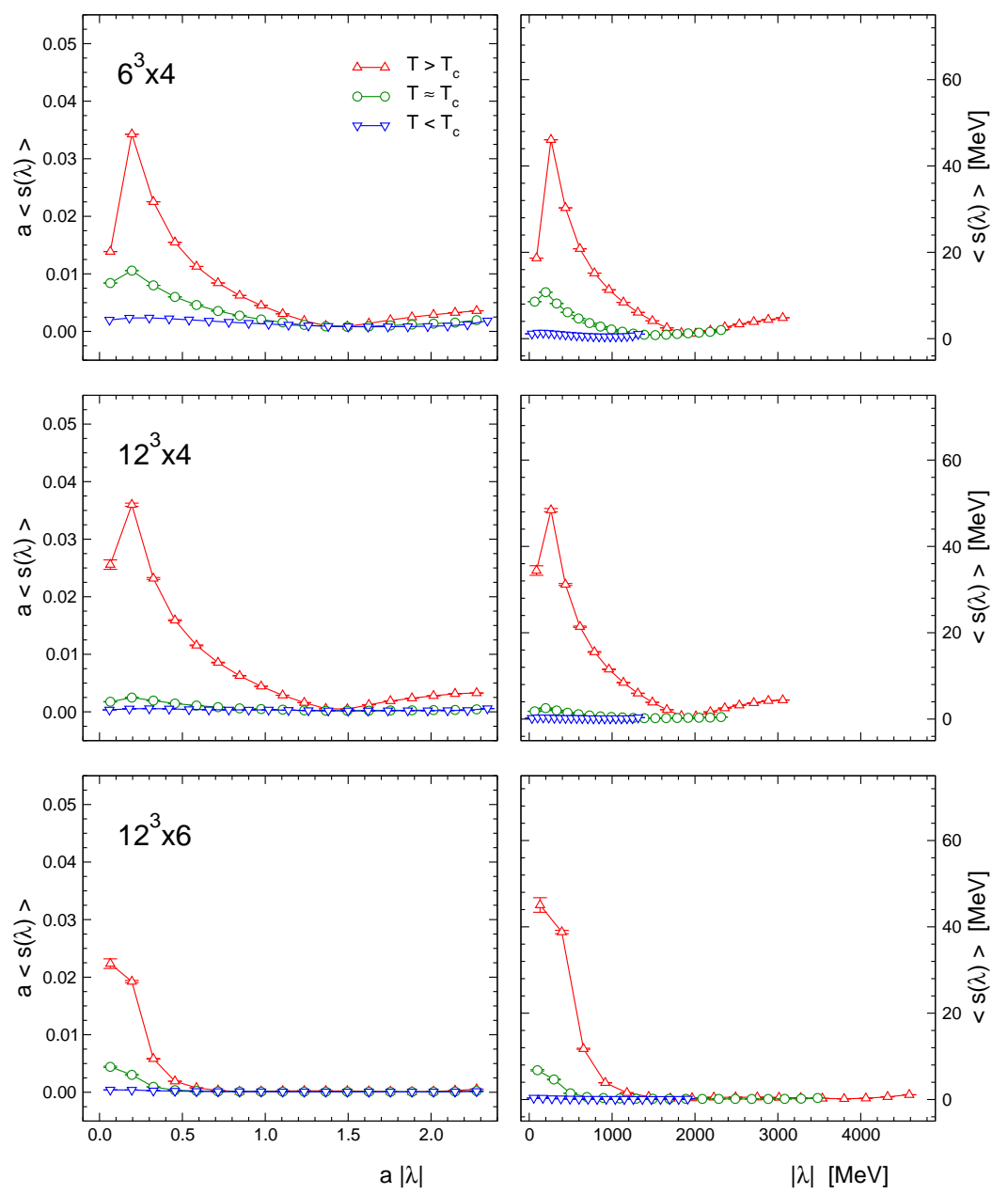

Figure 4: Average shift of the eigenvalues calculated as defined in Eq. (3.1) as a function of $\lambda$. Again we use lattice units in the 1.h.s. set of plots and $\mathrm{MeV}$ on the r.h.s.

and at $0.8 a$ and $1.9 a$ for $N_{t}=6$. It can be shown that these dips are correlated with a change of the direction of the shift of the eigenvalues for $z$-valued boundary conditions relative to the eigenvalues computed with periodic boundary conditions.

The plot in Fig. 5 shows that the individual contributions are biggest at the UV end of the spectrum. On the other hand, Fig. 3 shows that for the largest eigenvalues the density shows a sharp drop. It is an interesting question which effect, size of contribution versus density, wins out. This question can be addressed by considering only partial sums of (2.12). In Fig. 6, we show such partial sums as a function of the cutoff, i.e., as a function of the largest eigenvalue included in (2.12). Again we divide by the Polyakov loop $P$, such that when all eigenvalues are summed, i.e., at the largest value of $\lambda$ in Fig. 6, the curve approaches 1 .

For the $12^{3} \times 6$ lattice, we find that for low and medium temperatures the partial sums overshoot by nearly a factor of two before they reach the correct value at the largest values of $\lambda$. Thus the behavior of the partial sums is non-monotonic. On the other hand, for small temperatures the Polyakov loop should vanish. Thus the overshooting is not problematic, since the accumulated 

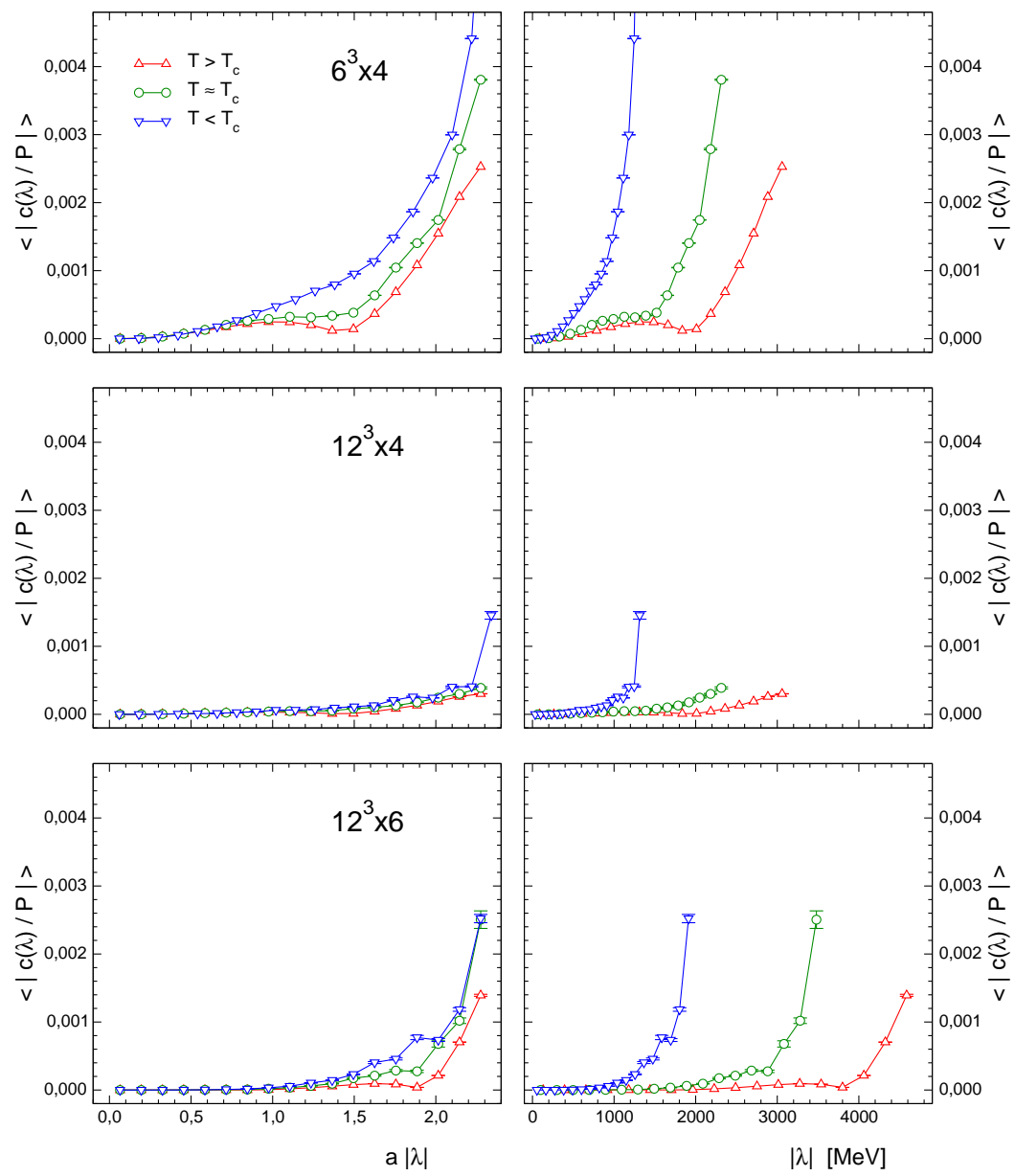

Figure 5: Contribution (3.2) of individual eigenvalues to the spectral sum for the thin Polyakov loop given by Eq. (2.12). As before we use lattice units in the 1.h.s. set of plots and $\mathrm{MeV}$ on the r.h.s.

contributions stay approximately zero over the whole range of the spectrum. We also find that the thin Polyakov loop is recovered only after all UV modes are included in the spectral sum. Again we see the appearance of dips at exactly the same values which we have already identified in Fig. 5.

So far we have only considered absolute values of quantities. However, the Polyakov loop and also the spectral sums are complex numbers and we can analyze their relative phase. In Fig. 7 we plot the angle $\Delta \phi$ between the truncated sums and the resulting thin Polyakov loop as a function of the cutoff used in the truncated sum. We show data for a $12^{3} \times 4$ and a $12^{3} \times 6$ lattice, both at $T>T_{c}$. We find that on the former lattice the accumulated contributions start with the wrong sign and recover the correct sign at the end. On the other lattice, although starting and ending with the correct sign, there is an intermediate region in which the truncated sum has the wrong sign. A comparison with lattices of different spatial size but the same temporal extent reveals, that this behavior depends only on the temporal extent.

A possible explanation for this phenomenon is the fact that the eigenvalues $\lambda$ of the staggered Dirac operator are purely imaginary. Thus $\lambda^{4 n}$ is a real positive number, while $\lambda^{4 n+2}$ is real but 

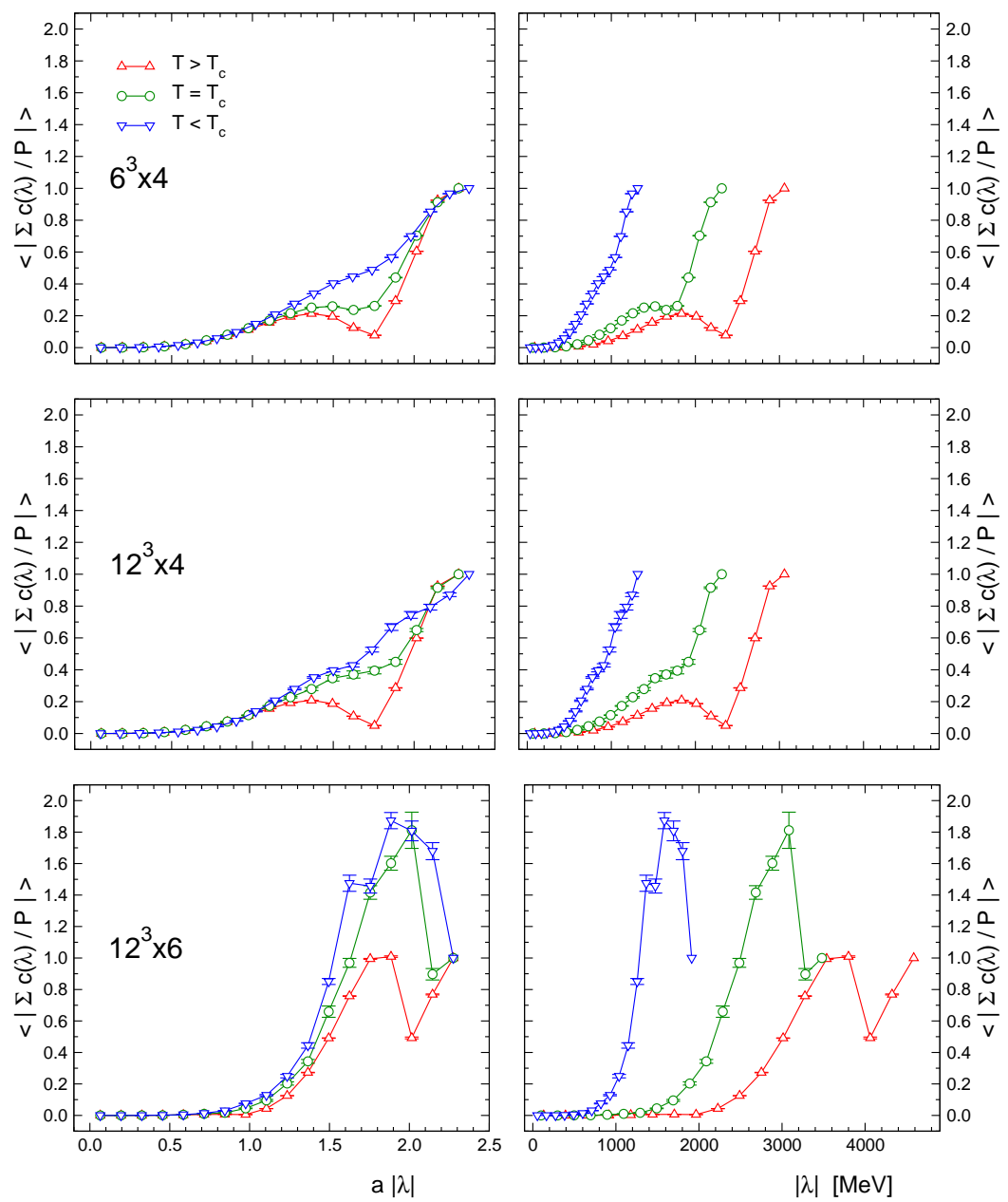

Figure 6: Partial sums of the spectral formula (2.12), plotted as a function of the cutoff.

negative. If we consider only the first term in the truncated sum we find:

$$
\begin{aligned}
& c\left(\lambda^{(1)}\right)=\frac{2^{N_{t}}}{V_{4}}\left[\left(\lambda^{(1)}\right)^{N_{t}}+z^{*}\left(\lambda_{z}^{(1)}\right)^{N_{t}}+z\left(\lambda_{z^{*}}^{(1)}\right)^{N_{t}}\right] \\
& =\frac{2^{N_{t}}}{V_{4}}\left[\alpha+z^{*} \tilde{\alpha}_{z}+z \tilde{\alpha}_{z^{*}}\right] \quad \stackrel{\tilde{\alpha}_{z^{*}} \approx \tilde{\alpha}_{z} \equiv \tilde{\alpha}}{=} \quad \frac{2^{N_{t}}}{V_{4}}\left[\alpha+\left(z^{*}+z\right) \tilde{\alpha}\right] \\
& =\frac{2^{N_{t}}}{V_{4}}[\alpha-\tilde{\alpha}]\left\{\begin{array}{ll}
<0 N_{t}=4 n & \text { as } \alpha, \tilde{\alpha}>0 \\
>0 N_{t}=4 n+2 & \text { as } \alpha, \tilde{\alpha}<0
\end{array},\right.
\end{aligned}
$$

where we assume that the boundary conditions with phases $z$ and $z^{*}$ lead to approximately the same lowest eigenvalue $\tilde{\alpha}$, which is larger in size than the eigenvalue at periodic boundary conditions $[9,5]$. Interestingly, this last relation between the relative sizes of the eigenvalues for non-trivial and periodic boundary conditions seems to change in certain regions in the spectrum of the staggered Dirac operator, thus creating the observed sign change in the truncated sums. The points where these sign changes occur depend only on the time extend of the lattice. 

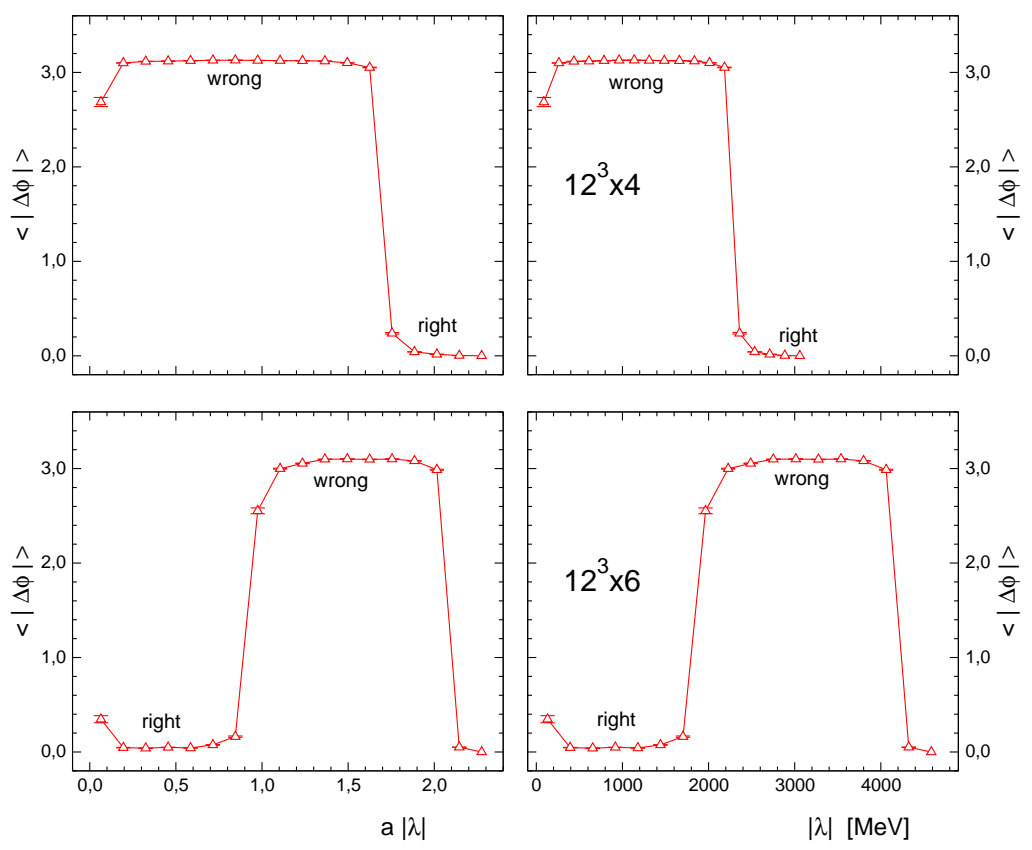

Figure 7: Phase shift of the truncated sums relative to the thin Polyakov loop for $T>T_{c}$. The upper plots are for a $12^{3} \times 4$ lattice, while the lower plots are for $12^{3} \times 6$.

\subsection{Dressed Polyakov loops}

For the thin Polyakov loops the numerical analysis of the last section demonstrates that the spectral sums for the thin loops are predominantly built up from the UV modes. However, as we have already speculated above, part of the UV dominance might come from the singular nature of the support of the thin loop. Thus it is interesting to perform the same spectral analysis for the dressed Polyakov loop. Such an analysis is planned for a future publication. Here we focus on the feasibility of computing the integral over the boundary angle in (2.19) numerically and establish that the dressed Polyakov loop is indeed an order parameter for the breaking of the center symmetry.

For the dressed Polyakov loops we compute complete spectra of $H$ for 16 values of the boundary angle $\varphi \in[0, \pi)$. The integrand in the spectral sum (2.19) is given by (omitting the normalization and the phase $\exp (-i \varphi q))$

$$
S(\varphi)=\frac{1}{V} \sum_{j=1}^{3 V} \frac{\kappa}{1-\kappa \lambda_{j}^{(\varphi)}} .
$$

Integrating $S(\varphi)$ over $\varphi$ with the phase $\exp (-i \varphi q)$ projects to the loops with winding number $q$. The important technical question is how smooth a function of $\varphi$ this integrand is.

Fig. 8 shows the integrand $S(\varphi)$ averaged over 20 configurations above $T_{c}$ with real Polyakov loops (l.h.s. plots) and 20 configurations with complex Polyakov loops (r.h.s.). The individual curves are for different values of the mass parameter $m$ in the vicinity of the critical mass $m_{c}$ where the Laplace operator ceases to be invertible. In the bottom plots a constant was subtracted. 

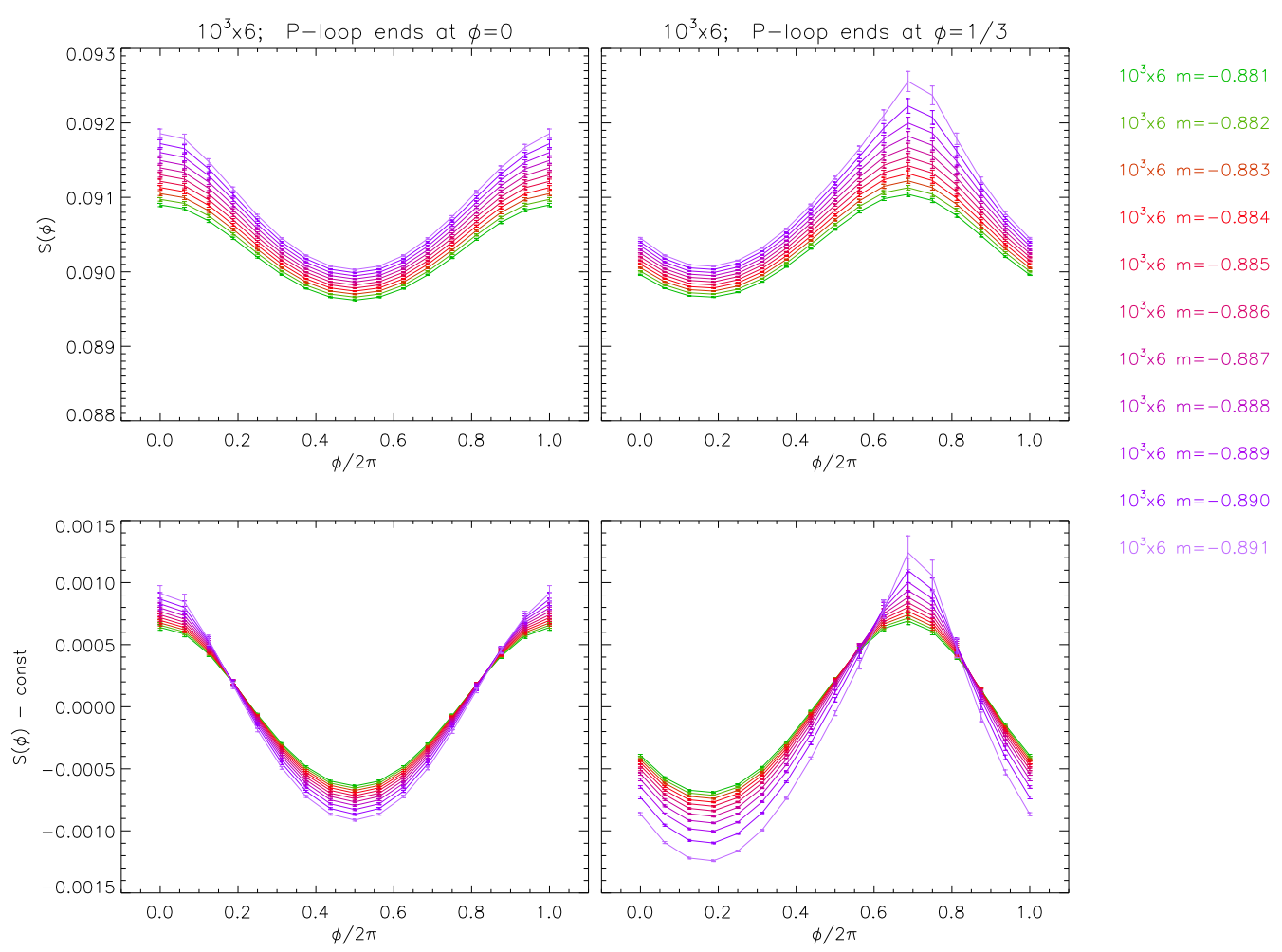

Figure 8: The spectral sum $S(\varphi)$ for the Laplace operator as a function of $\varphi$. The 1.h.s. plots are from an average over 20 configurations with real Polyakov loop, the r.h.s. plots are for 20 configurations with a Polyakov loop with phase $\sim \exp (i 2 \pi / 3)$. In the top plots the full sum $S(\varphi)$ is shown, while in the bottom plots the constant $c=\int d \varphi S_{L}(\varphi)$ was subtracted.

The plots show a clear cosine behavior for the ensemble with real Polyakov loops (l.h.s.) and a shifted cosine for the complex ensemble (r.h.s.). For low-lying modes a similar dependence on the phase at the boundary was observed in [10]. It is obvious that dividing the interval $[0, \pi)$ into 16 sub-intervals should be sufficient for a reliable numerical estimate of the $\varphi$-integral in the case of $q=1$, where the phase factor is $\operatorname{simply} \cos \varphi-i \sin \varphi$. This is the most interesting case of dressed Polyakov loops with a single winding.

Fig. 8 establishes that a numerical integration over $\varphi$ is feasible. We implement this integration for the case of $q=1$ using Simpson's rule. The resulting dressed Polyakov loop $P^{(1)}$ transforms under center transformations in the same way as the thin Polyakov loop $P$ defined in (2.1). Thus it should be an order parameter for breaking of center symmetry, but one even expects that it shows a similar behavior as shown in Fig. 1 for the thin loop. In order to test this expectation we use 20 configurations below $T_{c}$ and 20 above $T_{c}$. The latter all have a Polyakov loop which is essentially real, i.e., configurations in the right leg of the distribution shown in the r.h.s. plot of Fig. 1. On these two sets of configurations we compare the thin loop with the dressed loop at two different values of $\kappa$. The corresponding results in the complex plane are shown in Fig. 9.

The plot shows clearly that for $T<T_{c}$ both, the thin and the dressed Polyakov loops cluster around the origin, while above $T_{c}$ they develop a non-vanishing value near the positive real axis. Even the pattern in the distribution of the data points is similar for thin and dressed loops. 

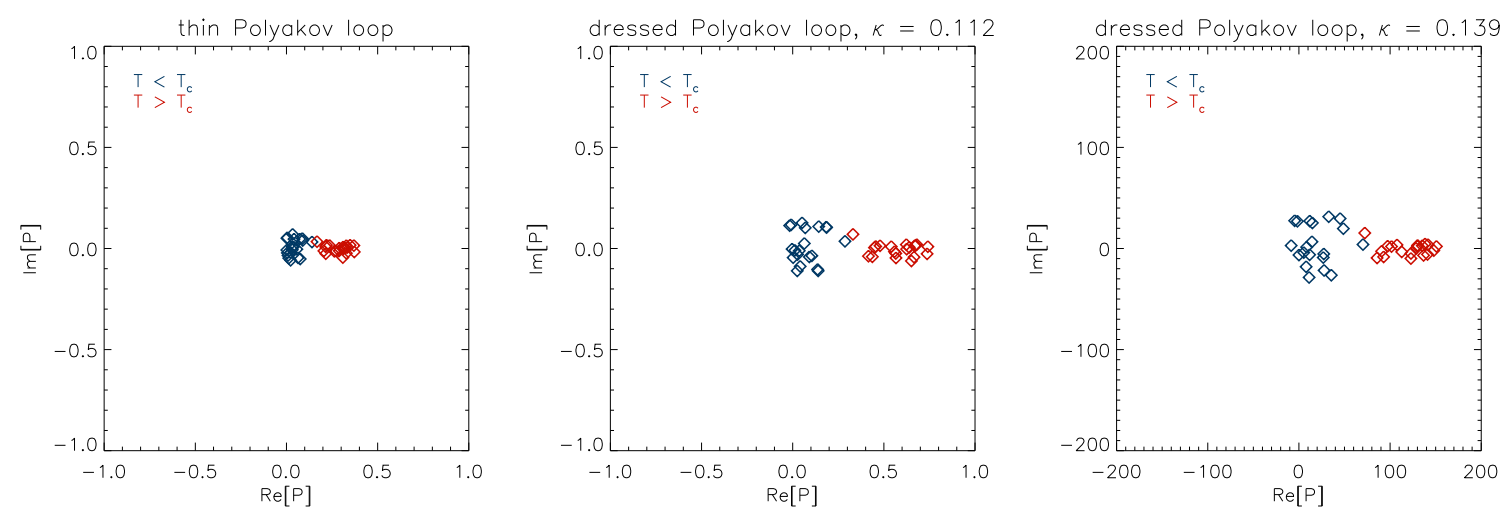

Figure 9: Scatter plots of thin (1.h.s. plot) and unscaled dressed Polyakov loops (center and 1.h.s. plots) below (blue symbols) and above $T_{c}$ (red symbols) for 20 configurations on a $12^{3} \times 6$ lattice.

The analysis of Fig. 9 shows that the dressed Polyakov loop (2.19) is a suitable order parameter for the breaking of center symmetry. It is less singular and is expected to have a proper continuum limit. We are currently analyzing the volume and temperature dependence of the dressed loops and will then return to studying which part of the spectrum predominantly contributes to the spectral sum for the dressed loops (2.19).

\section{Acknowledgments}

We thank Pierre van Baal, Tamas Kovacs, Kurt Langfeld, Wolfgang Söldner and Andreas Wipf for stimulating discussions. Erek Bilgici has been supported by Fonds zur Förderung der wissenschaftlichen Forschung (FWF DK W1203-N08) and by NAWI-Graz. Christian Hagen has been supported by BMBF.

\section{References}

[1] L. D. McLerran and B. Svetitsky, Phys. Rev. D 24, 450 (1981).

[2] T. Banks and A. Casher, Nucl. Phys. B 169, 103 (1980).

[3] C. Gattringer, Phys. Rev. Lett. 97, 032003 (2006) [arXiv:hep-lat/0605018].

[4] F. Bruckmann, C. Gattringer and C. Hagen, Phys. Lett. B 647, 56 (2007) [arXiv:hep-lat/0612020].

[5] F. Synatschke, A. Wipf and C. Wozar, Phys. Rev. D 75, 114003 (2007) [arXiv:hep-lat/0703018].

[6] W. Söldner, PoS(LAT2007) 222.

[7] M. Lüscher and P. Weisz, Commun. Math. Phys. 97, 59 (1985); Err.: 98, 433 (1985); G. Curci, P. Menotti, and G. Paffuti, Phys. Lett. B 130, 205 (1983); Err.: B 135, 516 (1984).

[8] C. Gattringer, R. Hoffmann, and S. Schaefer, Phys. Rev. D 65, 094503 (2002).

[9] T. Kovacs, private communication.

[10] F. Bruckmann and E. M. Ilgenfritz, Phys. Rev. D 72114502 (2005) [arXiv:hep-lat/0509020]; C. Gattringer and S. Schaefer, Nucl. Phys. B 654, 30 (2003) [arXiv:hep-lat/0212029]. 